\title{
Benchmark results in vector atmospheric radiative transfer
}

\author{
Alexander A. Kokhanovsky ${ }^{\mathrm{a}, *}$, Vladimir P. Budak ${ }^{\mathrm{b}}$, Celine Cornet $^{\mathrm{c}}$, Minzheng Duan ${ }^{\mathrm{d}}$, \\ Claudia Emde ${ }^{e, 1}$, Iosif L. Katsev ${ }^{f}$, Dmitriy A. Klyukov ${ }^{b}$, Sergey V. Korkin ${ }^{b}$, L. C-Labonnote $^{c}$, \\ Bernhard Mayer ${ }^{\mathrm{e}, 1}$, Qilong Min $^{\mathrm{g}}$, Teruyuki Nakajima ${ }^{\mathrm{h}}$, Yoshifumi Ota ${ }^{\mathrm{i}}$, \\ Alexander S. Prikhach ${ }^{f}$, Vladimir V. Rozanov ${ }^{a}$, Tatsuya Yokota ${ }^{i}$, Eleonora P. Zege ${ }^{f}$ \\ a Institute of Environmental Physics, University of Bremen, O. Hahn Allee 1, D-28334 Bremen, Germany \\ ${ }^{\mathrm{b}}$ Moscow Power Engineering Institute, Krasnokazarmennaya 14, Moscow 11250, Russian Federation

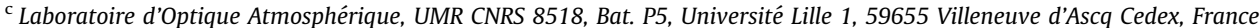 \\ d LAGEO, Institute of Atmospheric Physics, Chinese Academy of Science, Beijing 100029, China \\ e Deutsches Zentrum für Luft- und Raumfahrt (DLR), Münchner Straße 20, 82234 Weßling, Germany \\ ${ }^{\mathrm{f}}$ Stepanov Institute of Physics, National Academy of Sciences of Belarus, Pr. Nezavisimosti 68, 220072 Minsk, Belarus \\ ${ }^{\mathrm{g}}$ Atmospheric Science Research Center, State University of New York, Albany, NY 12203, USA \\ ${ }^{\mathrm{h}}$ Center for Climate System Research, University of Tokyo, 5-1-5 Kashiwanoha, Kashiwa-shi, Chiba 277-8568, Japan \\ ${ }^{i}$ Center for Global Environmental Research, National Institute for Environmental Studies, 16-2 Onogawa, Tsukuba 305-8506, Japan
}

\section{A R T I C L E I N F O}

\section{Article history:}

Received 18 November 2009

Received in revised form

9 March 2010

Accepted 9 March 2010

\section{Keywords:}

Radiative transfer

Aerosol

Clouds

Molecular scattering

Polarization

\begin{abstract}
A B S T R A C T
In this paper seven vector radiative transfer codes are inter-compared for the case of underlying black surface. They include three techniques based on the discrete ordinate method (DOM), two Monte-Carlo methods, the successive orders scattering method, and a modified doubling-adding technique. It was found that all codes give very similar results. Therefore, we were able to produce benchmark results for the Stokes parameters both for reflected and transmitted light in the cases of molecular, aerosol and cloudy multiply scattering media. It was assumed that the single scattering albedo is equal to one. Benchmark results have been provided by several studies before, including Coulson et al. [22], Garcia and Siewert [7,8], Wauben and Hovenier [10], and Natraj et al. [11] among others. However, the case of the elongated phase functions such as for a cloud and with a high angular resolution is presented here for the first time. Also in difference with other studies, we make inter-comparisons using several codes for the same input dataset, which enables us to quantify the corresponding errors more accurately.
\end{abstract}

(c) 2010 Elsevier Ltd. All rights reserved.

\section{Introduction}

The propagation of solar light in the terrestrial atmosphere, ocean, and vegetation is often treated in the framework of the scalar radiative transfer theory. However, due to the electromagnetic nature of light, the correct treatment of light reflection and transmission must include the effects of polarization. Exact analytical solu-

\footnotetext{
* Corresponding author.

E-mail address: alexk@iup.physik.uni-bremen.de (A.A. Kokhanovsky).

${ }^{1}$ Now at: Meteorologisches Institut, Ludwig-Maximilians-Universität, Theresienstr. 37, 80333 München, Germany.
}

tions for reflection and transmission functions exist for homogeneous plane-parallel media in the case when scattering effects can be neglected. Corresponding equations have been derived by Fresnel in 1823 [1,2] even before the electromagnetic nature of light was understood. The account for light scattering inside the medium brings us to the formulation and the necessity to solve the vector radiative transfer equation. It has the following form for the considered case of the illumination of a plane-parallel light scattering layer by a wide light beam $[3,4]$ :

$$
\mu \frac{d \vec{S}(\vec{\Omega})}{d \tau}=-\vec{S}(\vec{\Omega})+\frac{\omega_{0}}{4 \pi} \int_{4 \pi} \hat{P}\left(\vec{\Omega}^{\prime} \rightarrow \vec{\Omega}\right) \vec{S}\left(\vec{\Omega}^{\prime}\right) d \vec{\Omega}^{\prime}
$$


Here $\vec{S}(\vec{\Omega})$ is the Stokes vector at the optical depth $\tau$ in the direction specified by the vector $\vec{\Omega}, \omega_{0}$ is the single scattering albedo, $\mu$ is the cosine of the viewing zenith angle (VZA) $\vartheta$ and $\hat{P}\left(\vec{\Omega}^{\prime} \rightarrow \vec{\Omega}\right)$ is the phase matrix. Usually $\hat{P}\left(\vec{\Omega}^{\prime} \rightarrow \vec{\Omega}\right)$ and also $\vec{S}(\vec{\Omega})$ are defined with respect to the meridional plane containing the outer normal to the layer $\vec{n}$ and the propagation direction $\vec{\Omega}$. The components of the Stokes vector $\vec{S}(I, Q, U, V)$ in Eq. (1) are defined as follows [5]:

$I=\left\langle E_{\|} E_{\|}^{*}+E_{\perp} E_{\perp}^{*}\right\rangle, \quad Q=\left\langle E_{\|} E_{\|}^{*}-E_{\perp} E_{\perp}^{*}\right\rangle$,

$U=-\left\langle E_{\|} E_{\perp}^{*}+E_{\perp} E_{\|}^{*}\right\rangle, \quad V=i\left\langle\left(E_{\perp} E_{\|}^{*}-E_{\|} E_{\perp}^{*}\right)\right\rangle$

Here $E_{\|}$and $E_{\perp}$ are the projections of the electric vector on the directions parallel and perpendicular to the meridional plane containing the normal to the layer and the direction of propagation. The symbol $\langle>$ denotes an average over a time interval long compared with the typical period of fluctuations of the electromagnetic field. The averaging is needed because most of optical devices measure averages of radiation characteristics over a relatively long period of time. A common factor is omitted in Eq. (2). form:

Usually the Stokes vector is presented in the following

$\vec{S}=\vec{S}_{d i r}+\vec{S}_{d i f}$

Here

$\vec{S}_{d i r}=\vec{S}_{0} \delta\left(\cos \vartheta-\cos \vartheta_{0}\right) \delta\left(\varphi-\varphi_{0}\right) \exp (-\tau / \cos \vartheta)$

is the Stokes vector of the direct light and $\vec{S}_{d i f}$ is the corresponding vector for the diffuse light. The vector $\vec{\Omega}_{0}\left(\vartheta_{0}, \varphi_{0}\right)$ gives zenith $\vartheta_{0}$ and azimuth $\varphi_{0}$ angles of the incident light and $\vec{\Omega}(\vartheta, \varphi)$ specifies the viewing direction. $\vec{S}_{0}$ is the Stokes vector of incident light. We will assume that the incident light is unpolarized and, therefore, $\vec{S}_{0}=F_{0} \vec{J}$, where $\vec{J}^{T}=(1,0,0,0)$ is the unity vector and $F_{0}$ is the incident light irradiance at the area perpendicular to the incident light beam. The Stokes vector for the diffuse light can be found solving the following equation, which follows from Eqs. (1), (3), (4):

$$
\begin{aligned}
\mu \frac{d \vec{S}_{d i f}(\vec{\Omega})}{d \tau}=- & \vec{S}_{d i f}(\vec{\Omega})+\frac{\omega_{0}}{4 \pi} \int_{4 \pi} \hat{P}\left(\vec{\Omega}^{\prime} \rightarrow \vec{\Omega}\right) \vec{S}_{d i f}\left(\vec{\Omega}^{\prime}\right) d \vec{\Omega}^{\prime} \\
& +\frac{\omega_{0} F_{0}}{4 \pi} \hat{P}\left(\vec{\Omega}_{0} \rightarrow \vec{\Omega}\right) \vec{J} \exp \left(-\tau / \cos \vartheta_{0}\right)
\end{aligned}
$$

with boundary conditions specifying that there is no diffuse light entering the layer from the top and the bottom for the considered case of a medium with a black underlying surface and under the assumption that there is vacuum above the upper boundary of the layer, where the light source is located. There are numerous methods for the solution of the vector radiative transfer equation (VRTE). They include:

- Monte-Carlo method (MC);

- discrete ordinate method (DOM);

- successive-order-scattering method;

- doubling-adding,

to name a few (see, e.g., [20]). Different methods have different strong and weak points and great deal (as far as accuracy and the speed of calculations are concerned) depends on the chosen implementation of general and well-known equations. Although there are numerous papers on the solution of VRTE, the results are usually presented in plots and not in tables (especially in the cases when aerosol and cloud media are studied). This makes it difficult for new-comers to the fields to understand the accuracy and performance of their newly created software packages. With this in mind, we provide here the benchmark results of the VRTE solution, which can be used in future for the validation of the corresponding algorithms for the case of a vertically homogeneous plane-parallel light scattering nonabsorbing layer with black underlying surface. The benchmark results have been generated using SCIATRAN [6] and the differences in the final results obtained from those derived by other methods/codes are specified. Additional benchmark results can be found in the papers of Garcia and Siewert [7,8], Mishchenko [9], Wauben and Hovenier [10], and Natraj et al. [11].

\section{The description of codes}

\subsection{SCIATRAN}

SCIATRAN is aimed at the calculation of the Stokes vector-parameter $\vec{S}(I, Q, U, V)$ of reflected, transmitted and internal radiation from UV to the thermal IR $[6,12]$. The software package employs the discrete ordinates method (DOM) of the vector radiative transfer equation solution in a form very close to that given by Siewert [13]. The method of solution is based on the following steps:

- the phase matrix is presented as a series using generalized spherical functions;

- the Stokes vector is presented as a Fourier series with respect to the azimuth;

- the integration with respect to the azimuth is performed analytically;

- the integral with respect to the zenith angle is substituted by a series using the Gaussian quadrature rule;

- the system of differential equations derived in such a way is solved analytically (separately for cases of the single scattering albedo $\omega_{0}=1$ and $\omega_{0} \neq 1$ ); and

- the arbitrary constants in the analytical solution of the system of differential equations are found numerically using boundary conditions and solving the corresponding system of linear algebraic equations.

For vertically inhomogeneous media, the technique described above is applied to each sub-layer using boundary conditions describing the continuity of the solution.

In the simulations, it is assumed that the gaseous and condense matter absorption is negligible and the underlying surface is black. This was done to simplify the comparisons. As a matter of fact, SCIATRAN is equipped with HITRAN databases of the gaseous absorbers. Therefore, it can be used for the interpretation of hyperspectral 
measurements in the terrestrial atmosphere. Also it contains databases of local optical and thermal IR (till the wavelength $40 \mu \mathrm{m}$ ) characteristics of clouds and aerosols. Several models of underlying surfaces including land and ocean (with account of polarization) are implemented in the software package.

The components of the normalized Stokes vector $\vec{S}_{d i f}^{n}$ of the diffused light are obtained using the following relationship:

$\vec{S}_{d i f}^{n}=\left(\mu_{0} F_{0}\right)^{-1} \pi \vec{S}_{d i f}$

where $\mu_{0}$ is the cosine of the solar zenith angle (SZA) $\vartheta_{0}$ and $\vec{S}_{d i f}\left(I_{d i f}, Q_{d i f}, U_{d i f}, V_{d i f}\right)$ is the Stokes vector of the diffuse light. The viewing zenith angle $\vartheta$ is counted from the outer normal to the upper boundary. It varies from $0^{\circ}$ to $90^{\circ}$ for the reflected light and from $90^{\circ}$ to $180^{\circ}$ for the transmitted light.

The number of streams used in simulations and also the number of expansion coefficients to be accounted for are given in the SCIATRAN input file. To accelerate the calculations, the delta-M approximation is implemented in SCIATRAN. The software package with all databases can be freely downloaded after registration from the designated website www.iup.physik.uni-bremen.de/sciatran.

\subsection{Pstar}

The radiative transfer (RT) code, Pstar, has been developed to solve the vector RT equation for vertically inhomogeneous systems as approximated by several homogeneous layers [14]. The RT scheme of Pstar is constructed using the discrete ordinate method and the matrix operator method. The discrete ordinate method is applied to a single homogeneous layer in order to obtain the discrete ordinate solution, which consists of the reflection/transmission matrices and the source vector of the layer. To construct the multilayered system, the discrete ordinate solutions are obtained for each homogeneous layer; then the matrix operator method is applied to all layers to obtain the radiation field of the multilayered system.

This RT scheme is originally based on the formulations of Nakajima-Tanaka (N-T) [15] whose techniques are implemented in the scalar RT code series of System for Transfer of Atmospheric Radiation (STAR) [16]. By formulating the ground surface as a pseudo-layer in the matrix operator method, the discrete ordinate solution of the $\mathrm{N}-\mathrm{T}$ formulation is readily applicable to several different surface conditions. Recently, the N-T formulation was extended to express the polarized radiation field, which was implemented in the Pstar code [14]. Furthermore, the extended RT scheme is constructed to be flexible for a vertically inhomogeneous system including the internal oceanic layers as well as the ocean surface. Accordingly, the Pstar code is able to simulate the radiation field in the coupled atmosphere-ocean system including the polarization effect. The Pstar code is available from an internet web site of the OpenCLASTR (http://www.ccsr.u-tokyo.ac.jp/ clastr/) as well as the scalar RT code, Rstar, and is widely applicable to many problems, such as remote sensing data analysis, which require the account of the vector nature of light.

The Pstar consists of the radiative transfer code and an initialization code to calculate input parameters for the RT calculation on physical databases. The optical quantities of a layer and the number of discrete ordinate directions are adjustable depending on the problem to be solved. Given the scattering phase matrix, it is expanded into the azimuthally separated form using the generalized spherical functions. Then, the RT scheme of Pstar as described above computes the Stokes parameters at any interface between the homogeneous layers as well as at the top of the atmosphere. The Stokes parameters at any emergent direction are obtained by the post-processing of an analytical angular interpolation scheme. Finally, more accurate Stokes parameters are obtained using the truncated multiple+single (TMS) scattering correction procedure, which replaces the internal calculation of the single scattering contribution with the exact computation [17]. By the TMS procedure, Pstar is capable of performing accurate RT calculation efficiently with a small number of discrete ordinate directions, particularly for an optically thin atmosphere such as clear sky and background aerosol.

The Pstar computes all four Stokes parameters in the vector mode, although only the total radiance $(I)$ is obtained in the scalar mode. Furthermore, the semivector mode that computes the three Stokes parameters $(I, Q$ and $U$ ) on the basis of the $3 \times 3$ phase matrix approximation is available because the Stokes parameter $V$ is often much smaller than the other three parameters and almost negligible in the case of Mie scattering media. Also it follows that $V \equiv 0$ for the cases of molecular scattering and for the Mie scattering in exactly perpendicular observation (or incidence) geometries. In the latter case also the parameter $U$ vanishes and light is linearly polarized either in the plane perpendicular or parallel to the meridional plane. The degree of linear polarization is calculated as $p_{l}=-Q / I$ (at $U=0$ ) giving positive numbers for the case of molecular scattering.

Because the computation of eigensolutions of the discrete ordinate method is time consuming, the Pstar uses the other approach for obtaining the eigensolutions as compared to that used in the scalar code Rstar. In the vector mode, the direct decomposition method [14] is used in order to acquire the complex eigensolutions, which is necessary to calculate the Stokes parameter $V$ accurately. However, in the scalar and semi-vector modes, a squareroot decomposition technique as described by Nakajima and Tanaka [15] is invoked to obtain the real eigensolutions efficiently. For the inter-comparison of RT codes, the vector mode was used for computing four Stokes parameters.

\section{3. $R A Y$}

The radiative transfer code $R A Y$ has been developed to solve the VRTE for a vertically inhomogeneous light scattering, absorbing, and emitting plane-parallel layer with external light sources. The vertically inhomogeneous layer is approximated by a large number of homogeneous layers. The reflection surfaces are included as 
pseudo-layers. The layers with different refraction indices can be introduced at any level. Particularly, RAY allows the computation for the coupled systems such as atmosphereunderlying surface (e.g., an atmosphere-ocean system, both with the ocean plane and wind-roughened surfaces). The Green's matrix and all Stokes components can be computed at any point of the stratified system.

The VRTE is formulated for the Green's matrix of the problem that allows the simultaneous computations for incident light with any state of polarization and for a series of incident and observation angles [18]. The twocomponent approach [19] is used to reduce the computation time. Under this approach the phase function $F_{11}(x)$ (that is the $(1,1)$ element of the single scattering matrix) is represented as a sum of two components with the first component $F_{11}^{f}$ being different from zero only at small scattering angles $\theta \leq \theta_{\lim } \sim 10^{\circ}$. Correspondingly, the total phase and Green's matrices are also presented by the sums of two components. Two different radiative transfer problems for the two components $\mathbf{G}^{f}$ and $\mathbf{G}^{d}$ of the Green's matrix $\left(\mathbf{G}=\mathbf{G}^{f}+\mathbf{G}^{d}\right)$ are formulated instead of the original one.

The first component $\mathbf{G}^{f}$ of the solution satisfies the independent VRTE with the small-angle phase matrix. The second component of the Green's matrix $\mathbf{G}^{d}$ satisfies to the VRTE problem with the diffuse source defined through the solution of the first equation. The first equation is defined only at small scattering angles. The corresponding single scattering albedo is about 0.5 in this case. Therefore, it can be solved within the semi-analytical small-angle approach $[19,20]$. The solutions of the second equation are smoother functions as compared to the solutions of the initial RT equation, which enables a much simpler computation by any classical method [21]. In particular, the adding-doubling technique is currently implemented in $R A Y$. Using theory developed by Zege and Chaikovskaya [18], the VRTE for the matrix $\mathbf{G}^{f}$ is split into independent scalar-like small-angle equations, which are solved analytically using the small-angle approximation [20].

The additional time saving in the $R A Y$ code is achieved due to the use of

(a) the symmetry and reciprocity relationships in the process of computations of the Fourier harmonics in the adding-doubling technique;

(b) the analytical asymptotic approximations for a sum of the multi-dimensional integrals describing the rereflections between thick layers in the doubling procedure;

(c) the analytical description of the behavior of the Green's matrix elements in the vicinity of points $\mu=1$ and $\mu_{0}=1$.

Note that the Green's matrix components for singly scattered radiation are calculated without making any approximation.

The angle $\theta_{\text {lim }}$ (see above), the number of the Legendre polynomials in the expansion of the phase function (respectively, the number of the generalized spherical functions in the expansions of the single-scattering matrix elements) and the order of Gaussian quadratures are adjustable. Any accuracy required by the problem under consideration can be achieved with this code at the expense of increased computation time. The $R A Y$ version currently used is a Win32 application that runs on an ordinary PC. That limits the order of Gauss quadratures to 81. The version used in this paper for comparisons was optimized for the satellite data processing, where processing time is crucial. The LINUX version that is under development at the moment will allow any choice of adjustable parameters and will provide any required accuracy for any scattering media. Shortly RAY code will be accessible by contacting the authors (e.g., via e-mail: eleonor@light.basnet.by).

Previous estimations demonstrated high efficiency of the $R A Y$ code. For instance, the reflection and transmission matrices for the light scattering layer with just molecular scattering computed with the $R A Y$ code coincide within 5 digits with the tabular data [22]. The excellent coincidence of $R A Y$ results with SCIATRAN computations was obtained for the case of aerosol media [23]. The comparison of the RAY computations with the advanced Monte-Carlo code for the atmosphere-ocean system demonstrated the exemplary agreement [24]. As numerous comparisons with other codes have shown, $R A Y$ achieves an accuracy similar to that of others codes and provides highly accurate data in a fraction of the time required by the Monte-Carlo and other methods [24].

Thus, the $R A Y$ code does not require the use of very powerful computers; it runs quickly on an ordinary personal computer. The RAY code provides the tradeoff between time and accuracy of computations and allows one to achieve practically any accuracy at the expense of computation time. Its strength is in the simultaneous use of the advanced numerical and analytical results of modern radiative transfer theory. The mode, which is based on pure adding-doubling procedure (without any approximations) is also implemented in the $R A Y$ software package.

\subsection{MYSTIC}

The radiative transfer model MYSTIC (Monte-Carlo code for the phYSically correct Tracing of photons In Cloudy atmospheres) [25-27] is one of the most versatile Monte-Carlo codes for atmospheric radiative transfer. It is operated as one of several radiative transfer solvers of the libRadtran radiative transfer package [28], which is freely available at www.libradtran.org. MYSTIC calculates polarized solar and thermal radiance, irradiance, and actinic flux. The model has been used for remote sensing as well as for climate and photochemistry applications. MYSTIC allows the definition of arbitrarily complex 3D clouds and aerosols, inhomogeneous surface albedo and topography. To describe reflection at the surface a reflectance matrix can be defined. In 1D mode, the model can be operated in fully spherical geometry, which is important for limb sounding applications. MYSTIC can be run in forward and in backward tracing modes.

In order to include polarization accurately and at the same time efficiently, several Monte-Carlo methods have 
been combined. The local estimate method $[29,30]$ has been adapted to account for polarization. This method allows to compute radiances accurately using a reasonable number of photons. Furthermore an importance sampling method is used to sample the photon direction after a scattering event [31]. Whereas in the scalar mode the direction depends on the scattering zenith angle only, it depends also on the azimuth angle in the polarized mode. New variance reduction techniques have been developed to reduce the statistical noise in case of strongly peaked scattering phase functions that are typical of clouds and large aerosol particles [32]. Compared to the scalar mode, MYSTIC requires about 20\% more computational time for an aerosol optical thickness of about 0.2 , for larger aerosol optical thickness and for clouds the computation time may double.

The polarized model has been validated by comparison against benchmark results $[11,22,10]$. The relative differences were not systematic and below $0.5 \%$, which is well within the Monte-Carlo noise. Model results have also been compared to ground-based polarized radiance measurements [33]. The agreement was within the measurement uncertainty when an appropriate aerosol model was used as input.

\subsection{DMCPOL}

3DMCPOL is an atmospheric radiative transfer model computing the polarization state of the light in inhomogeneous three-dimensional light scattering media [34]. The code performs monochromatic calculations limited to solar wavelength range, which means that scattering and absorption processes are included but not thermal emission. It computes the upward and downward flux and the complete Stokes vector $(I, Q, U, V)$ and hence allows the computation of the total and polarized reflectances for an atmosphere composed of clouds, aerosols and molecules for various viewing zenith and azimuthal angles.

The model follows the general scheme of atmospheric forward Monte-Carlo [25] and uses at each scattering event the local estimate method [30] in order to reduce the statistical noise and, therefore, the number of photons required. The medium is described in a 3D Cartesian grid. Inputs for each grid cell are the extinction coefficient, the single scattering albedo and the phase matrix. Indeed, contrary to many other methods, the phase matrix is directly used in the calculation. So we avoid decomposition in Legendre polynomials that can require a large number of coefficients to describe accurately elongated phase functions. Concerning ground reflections, these are only considered as Lambertian and accordingly no treatment of the polarization modification by the surface is done.

Comparing to the scalar approach, the vector approach implies two specificities. First, during a scattering event the scattering azimuth angle is not chosen uniformly between $0^{\circ}$ and $180^{\circ}$ but depends on the scattering zenithal angle and on the polarization state of the incident beam. A conditional probability density function is computed for the determination of the azimuthal angle. The phase matrix, which describes the change of the incident Stokes vector after a scattering event, is defined in the scattering plane while the photon travel is followed in the meridian plane. Two appropriate rotations are thus performed between the meridian and the scattering plane and conversely [3]. These additional operations lead to an increase of the calculation time by a factor of about two. A comprehensive description of the code is given by Cornet et al. [34,35].

\subsection{SOSVRT}

SOSVRT is a polarized radiative transfer model based on successive orders of scattering [36,37]. When solar light enters the atmosphere, it undergoes processes of scattering and absorption. The scattered light becomes partially polarized. Photons could be scattered once or even hundreds times, depending on the optical properties of the scattering media. The total number of photons reaching the detectors can be given by summation of all orders of scattering. Then it follows for the Stokes vector:

$\vec{S}(\tau, \mu, \phi)=\sum \vec{S}_{n}(\tau, \mu, \phi), \quad n=1,2,3, \ldots \ldots \infty$

Here ' $n$ ' stands for the order of scattering and $\phi=\varphi-\varphi_{0}$ is the relative azimuthal angle (RAA). For simplicity, Fourier decomposition is made for the Stokes vector and also for the scattering phase matrix. Then the numerical solution is obtained for each Fourier component. The analytical method of decomposition of the phase matrix developed by Siewert [38] is used in SOSVRT, which is based on generalized spherical functions and can be given recursively. Moreover, the rotation of the coordinate system is taken into account. This way has been proved to be efficient and accurate and has been successfully implemented into various vector radiative transfer codes (see above). To further improve the efficiency of Fourier decomposition, three symmetry relationships for the respective Mueller matrix $\mathbf{M}$ [24] of the light scattering layer are implemented:

$\mathbf{M}\left(-\mu_{i},-\mu_{j}\right)=\mathbf{E M}\left(\mu_{i}, \mu_{j}\right) \mathbf{E}$
$\mathbf{M}\left(\mu_{i}, \mu_{j}\right)=\mathbf{E}_{1} \mathbf{M}^{T}\left(\mu_{j}, \mu_{i}\right) \mathbf{E}_{1}$
$\mathbf{M}\left(\mu_{i},-\mu_{j}\right)=\mathbf{E}_{2} \mathbf{M}^{T}\left(\mu_{j},-\mu_{i}\right) \mathbf{E}_{2}$

where $\mathbf{E}=\operatorname{diag}\{1,1,-1,-1\}, \mathbf{E}_{\mathbf{1}}=\operatorname{diag}\{1,1,-1,1\}$, $\mathbf{E}_{2}=\operatorname{diag}\{1,1,1,-1\}$. The first two relationships are, respectively, relationships $G$ and $E$ given by Hovenier [39], and the third relationship is derived from the first two relationships. If the grid points are symmetric, 3/4 of the time will be saved in calculation of the Muller matrix of the light scattering layer if Eqs. (8) are used.

The solution for each Fourier component and each order of scattering is given by the integration of the radiative transfer equation along the line of sight, where the single scattering could be accurately described by the analytical formula. Hundreds of orders of scattering may be needed to ensure the convergence, particularly for the single scattering albedo values close to 1.0 , optically thick media, oblique incidence and observation angles. Therefore, the successive order of scattering method is often 
thought to be inefficient. To improve the computational efficiency, a simple approximation technique is used. It is based on the truncating of scattering orders with the following series:

$S_{j}(\tau, \mu, \phi)=\sum_{n=1}^{N-1} S_{j, n}(\tau, \mu, \phi)+S_{j, N}(\tau, \mu, \phi) /\left(1-c_{j}\right)$

It is assumed that $c_{j}=S_{j, N+1} / S_{j, N}$, where $S_{j}$ is the $j$ th component of the Stokes vector. This is a very accurate approximation due to the fact that the photons lose memory of their origin after several scattering events and radiation approaches a diffusion regime.

To improve the accuracy, a post-processing procedure is used. It interpolates and extrapolates Stokes vectors to arbitrary angles analytically by re-calculation of the source function at user defined angles. This procedure is similar to that of Nakajima and Tanaka [17] but with polarization. Also the truncated multiple+single scattering technique is used [17]. With these speeding-up techniques applied, the SOSVRT shows great advantages in computation efficiency compared with other vector algorithms, particularly for optically thin atmosphere such as clear sky and cirrus clouds whose optical depth is often less than 4 . For optically thick media such as extended water clouds, which require large number of streams to approach accurate results, SOSVRT also shows great advantage in accuracy and efficiency.

By tracing the photons for each scattering event, SOSVRT provides physical insights in radiation absorption processes when scattering evens take place. Moreover, the vertical variation of atmospheric optical properties such as absorption and scattering can be easily taken into account. SOSVRT is written in FORTRAN 90 and code is freely accessible by contacting the authors (e.g., via e-mail: dmz@mail.iap.ac.cn).

\subsection{MVDOM}

The radiative transfer code MVDOM (Modified Vectorial Discrete Ordinates Method) has been developed to solve the vector radiative transfer equation for the case of a vertically inhomogeneous light scattering and absorbing plane-parallel layer. The layer is assumed to be horizontally homogeneous and illuminated by a collimated light beam at an arbitrary angle. The polarization state of an incident beam is assumed to be arbitrary. The MVDOM code computes all four Stokes-vector parameters for the diffusely reflected and transmitted light. The phase matrix is assumed to be block-diagonal one. Aerosol scattering, cloud scattering, Rayleigh scattering and an arbitrary combination of the mentioned types of scattering can be used in calculations. Gamma, lognormal or Junge distribution are used as particles size distribution functions for Mie scattering. The expansion of the phase matrix elements with respect to generalized spherical functions is provided by initialization code. The resulting coefficients are written to a data-file and used further on without re-calculation unless the phase matrix is changed.

Provided a high degree of scattering anisotropy of the real media, the radiative transfer scheme of MVDOM is constructed upon the representation of the VRTE solution as the superposition of the most anisotropic (Singular-S) part and smooth regular $(R)$ parts [40]. The former is calculated upon the vectorial modification of spherical harmonics method (MSH) derived upon the smoothness of the solution's spatial spectrum $[41,42]$ after the desired solution is expanded with respect to generalized spherical functions [47]. The MSH gives the approximate solution of the VRTE. It describes the most anisotropic part of the VRTE solution and it contains the solution's singularity caused by ray approximation. Regular part is evaluated from a VRTE boundary problem with a new source function using the MSH. Discrete ordinates method [13] is used to obtain the system of differential equations, which is solved then.

The vertical inhomogeneity is included by means of matrix operator method [43] for hemispheres [44] but with a new vector source function.

The ill-conditionality for arbitrary thickness is avoided by scaling transformation [45] when the eigenvectors and eigennumbers problem is solved. Scaling transformation allows MVDOM to compute Stokes vector for systems with an arbitrary thickness ( $\tau=500$ and more). The boundary conditions are formulated in the form of Mark [46]. The solutions are given in the matrix formulation with all matrices defined analytically.

The significant smoothness of the regular part leads to the small number of azimuthal harmonics (below 6) need to be accounted for (even for the inclined illumination at angle $80^{\circ}$ or so).

The matrix form of the solution makes The MathWorks Matlab interface very convenient to write the computational routine. The MSH as a source function does not change principally the mathematical form of the VRTE boundary problem. So all known methods (discrete ordinates method, spherical harmonics method and so on) can be used to obtain the solution. The calculation time for a typical case is some seconds using standard PC and less then one second for Rayleigh scattering. The scalar approximation for the VRTE solution based upon DOM, matrix operator method and scalar transformation is also available for a multi-layered system.

\section{The definition of cases for the inter-comparison}

The inter-comparisons of the codes described above have been performed for the case of nonabsorbing homogeneous plane-parallel light scattering layers with black underlying surface for three values of the relative azimuth $\left(0^{\circ}, 90^{\circ}\right.$, and $180^{\circ}$ ) and the solar zenith angle equal to $60^{\circ}$. The case of $\mathrm{RAA}=180^{\circ}$ corresponds top the exact backscattering direction at $\vartheta=\vartheta_{0}$. Three cases have been considered:

- pure Rayleigh scattering,

- aerosol layer,

- cloud.

The optical thickness of the cloud layer was equal to 5 and the optical thickness of molecular atmosphere was assumed to be equal to 0.3262 , which correspond to the molecular optical thickness of terrestrial atmosphere at 
the wavelength $\lambda=412 \mathrm{~nm}$. The optical thickness of an aerosol layer was set to 0.3262 as well.

The Rayleigh scattering matrix was taken in the standard form (without depolarization factor). The scattering matrices of aerosol and cloud media have been calculated using the Mie theory at $\lambda=412 \mathrm{~nm}$. They are presented in Fig. 1 and also corresponding numerical values are given at www.iup.physik.uni-bremen.de/ alexk. It was assumed that the radii $a$ of aerosol and cloud particles follow the lognormal distribution

$f(a)=\frac{1}{\sqrt{2 \pi} s a} \exp \left(-\ln ^{2}\left(a / a_{0}\right) / 2 s^{2}\right), \quad \int_{0}^{\infty} f(a) d a=1$

with $a_{0}=0.3 \mu \mathrm{m}, s=0.92, a_{\text {end }}=30 \mu \mathrm{m}$ in the case of aerosol medium and $a_{0}=5 \mu \mathrm{m}, s=0.4, a_{\text {end }}=100 \mu \mathrm{m}$ for a cloud. The value of $a_{\text {end }}$ specifies the upper limit of integration of Mie optical cross sections with respect to the radius of particles $a$. The lower limit of integration was equal to $0.005 \mu \mathrm{m}$. The refractive index of aerosol particles $m$ was set to 1.385 and it was assumed that $m=1.339$ for cloud

a

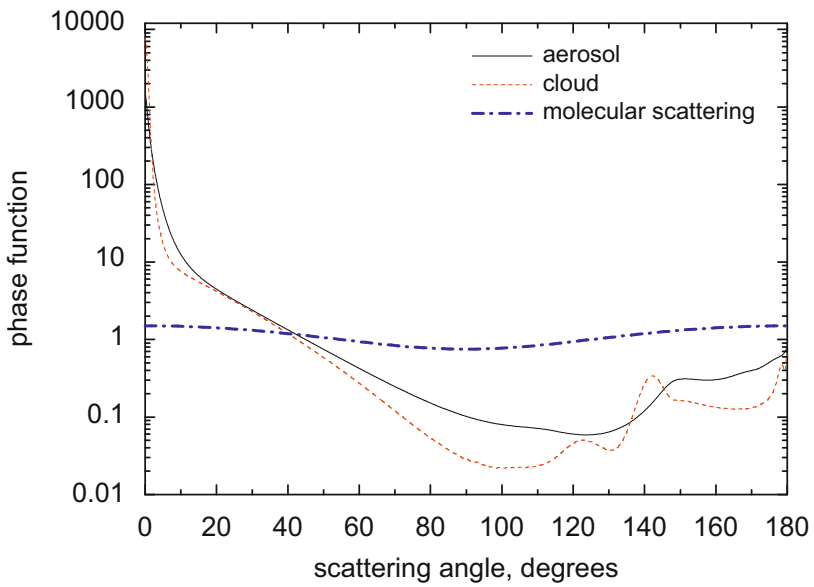

b

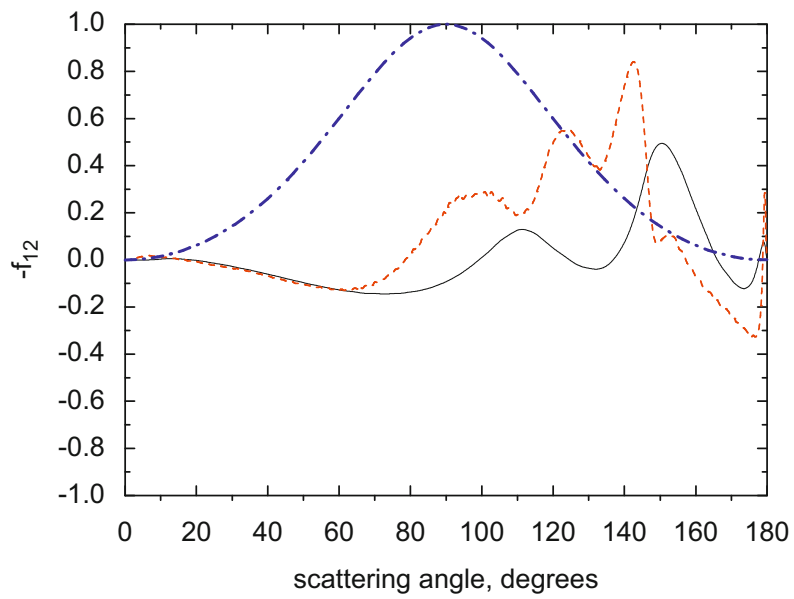

droplets. The calculated asymmetry parameters were equal to 0.79275 and 0.86114 for aerosol particles and water droplets, respectively. Therefore, both phase functions are highly elongated in the forward direction, which is typical for atmospheric aerosols and clouds.

The droplet size distribution is quite narrow $(s=0.4)$. Therefore, the rainbow and glory are clearly seen in Fig. 1a for the cloud phase function. These oscillations are especially difficult to handle with some types of radiative transfer codes. Other elements of the scattering matrix also oscillate as a function of the scattering angle. The degree of linear polarization of singly scattered solar light is given by the element $-f_{12}$ shown in Fig. 1b. It follows that the degree of polarization is positive in the rainbow region for clouds. The degree of polarization of molecular scattering is positive and, therefore, oscillations occur predominantly in the plane perpendicular to the scattering plane. Summing up, the scattering matrices of aerosol and especially cloudy media are selected in such a way that they can pose some difficulties for radiative transfer codes. The idea behind the selection of these cases was to
C

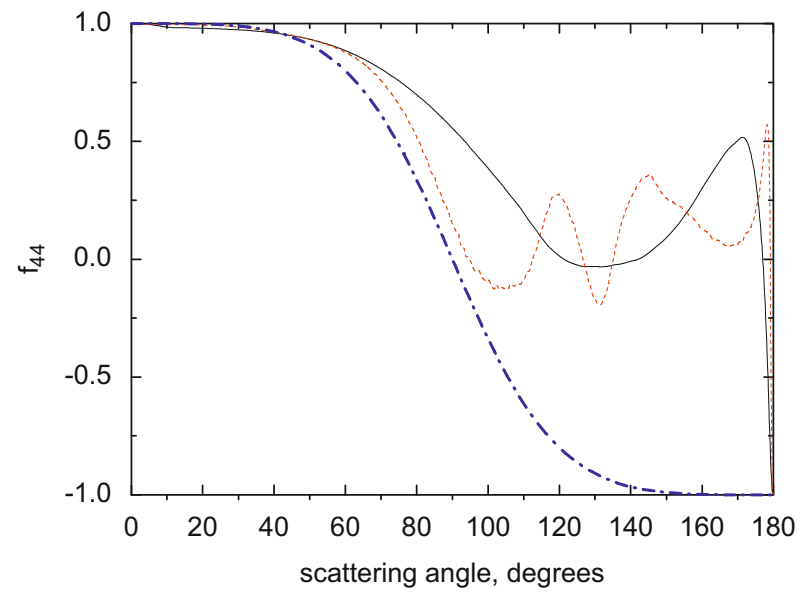

d

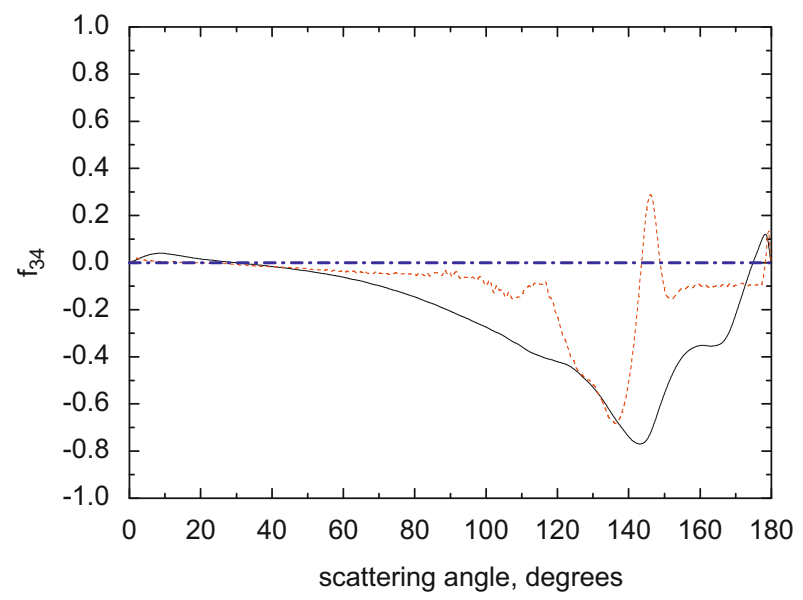

Fig. 1. (a) Phase functions normalized as $\int_{0}^{\pi} p(\theta) \sin \theta d \theta=2$ for the models used; (b) the degree of polarization for the models used ( $-f_{12} \equiv-F_{12} / F_{11}$, where $F_{i j}$ are elements of the scattering matrix); (c) the same as in (a) except for $f_{44} \equiv F_{44} / F_{11}$; and (d) the same as in (a) except for $f_{34} \equiv F_{34} / F_{11}$. 
see the performance of the individual radiative transfer codes in extreme situations.

\section{Results}

In this work, the normalized Stokes parameters defined as

$I_{R}=\pi I_{d i f} / \mu_{0} F_{0}, \quad Q_{R}=\pi Q_{d i f} / \mu_{0} F_{0}, \quad U_{R}=\pi U_{d i f} / \mu_{0} F_{0}$,

$V_{R}=\pi V_{\text {dif }} / \mu_{0} F_{0}$

(and similarly for the transmitted light (e.g., $\left(I_{T}, Q_{T}, U_{T}, V_{T}\right)$ ) are calculated and inter-compared. The results of calculations using the DOM codes (SCIATRAN, Pstar) and MYSTIC code are shown in Fig. 2 for the case of Rayleigh scattering, $\mu_{0}=0.5$ and relative azimuths $0^{\circ}, 90^{\circ}$, and $180^{\circ}$. The same dependencies on the viewing zenith angle have been obtained for all other codes. We found that all above mentioned codes can handle the case of Rayleigh scattering without any problem. The case of aerosol scattering is also handled well by all codes in a correct way (see Fig. 3). The MC method (at used number of photons) becomes less accurate for very small radiance values. In particular, the fourth Stokes vector component (and also the third component as $\vartheta \rightarrow 90^{\circ}$, see Fig. 3 ) is computed with large errors then. The component $V$ is usually of no use in remote sensing and, therefore, all three codes can be used for the processing of data related to remote sensing experiments for the case of a cloud-free atmosphere. Note that the accuracy of MC method can be increased if more photons are used in calculations. The accuracy of the DOM method can be increased if more streams are taken into account. For the aerosol scattering SCIATRAN used 240 streams (480 Legendre coefficients) in the hemisphere and the Pstar code used 180 streams and 360 Legendre coefficients. It follows from the analysis of Fig. 3 that the increase in number of streams by a factor of 1.5 does not influence the results of calculations in a considerable way-although the speed of calculations decreases considerably then. Actually, although the number of streams should be tuned depending on the problem to be solved, the high accuracy for satellite remote sensing data analysis is obtainable with fairly-low number of streams for most geometries. The extremely large number of streams was used in this study in order to show more accurate results for all cases (especially for the region of small scattering angles and also for the fourth Stokes parameter).

The case of a cloud layer is considered in Fig. 4. For this case Pstar has used 180 streams (360 Legendre coefficients) and SCIATRAN used 360 streams (720 Legendre coefficients). Again the results were almost the same, which meant the differences between 180 and 360 streams were not significant even for this case. Here, the extremely large number of streams was used for the same reason as for the aerosol case.

The accuracy of the MC method (for the selected number of photons, $10^{8}$ ) is not sufficient to describe the fourth element of the Stokes vector, which is smaller than $10^{-4}$. Otherwise, it provides accurate values of $(I, Q U)$ parameters for a cloud both in reflected and transmitted light.
It follows from the comparison of results given in Figs. 2-4, that the reflected light intensity increases towards large viewing zenith angles at the relative azimuthal angle equal to zero degrees in the considered case of the solar zenith angle equal to $60^{\circ}$. This is due to the fact that the scattering angle $\theta$ decreases from $120^{\circ}$ to about $30^{\circ}$ as the viewing zenith angle changes from $0^{\circ}$ to $89^{\circ}$ (at $\phi=0^{\circ}$ ). The phase function increases in this case (see Fig. 1). Similar effect takes place at $\phi=90^{\circ}$ but then the increase of $\theta$ is not so pronounced (just from $120^{\circ}$ to $90^{\circ}$ ) and, therefore, the corresponding peaks are lower. The case of $\phi=180^{\circ}$ corresponds to the backscattering geometry (at $\vartheta=\vartheta_{0}$ ). Therefore, the glory peaks are seen in dependencies of $I_{R}(\vartheta)$ both for aerosol and cloud scattering phase functions in this case. The rainbow feature is also clearly seen in the functions $I_{R}(\vartheta)$ and $Q_{R}(\vartheta)$ for the cloud case. The dependence $I_{R}(\vartheta)$ for the Rayleigh scattering is quite smooth and this is because the phase function does not have a sharp peak in the forward scattering direction.

The analysis of figures for transmitted light confirms that all codes are capable of describing the forward scattering peak in a correct way, which usually is a challenge for the strongly peaked phase functions. The Rayleigh scattering case does not produce any peak (see Fig. 2). Although the dependence of $Q_{T}(\vartheta)$ is quite smooth for Rayleigh scattering, there are oscillations in the value of $Q_{T}(\vartheta)$ close to the forward scattering direction both for cloud and aerosol media. The value of $Q_{T}$ is smaller for the cloud as compared to the aerosol. This is due to the multiple scattering effects, which are stronger for the cloud (optical thickness $\tau_{0}=5$ ) as compared to the aerosol medium $\left(\tau_{0}=0.3262\right)$. The differences in characteristics of reflected and transmitted light for aerosol and Rayleigh scattering are exclusively due to different phase matrices because $\tau_{0}$ and $\omega_{0}$ are the same for both cases. Note that the fourth Stokes component is exactly zero for the Rayleigh scattering case and it vanishes at relative azimuthal angles $0^{\circ}$ and $180^{\circ}$.

\section{Discussion and summary}

Seven radiative transfer codes have been compared in this work. The results obtained for the case of molecular and aerosol scattering were almost identical for the discrete ordinates codes developed by independent teams (SCIATRAN and Pstar). Very similar results have also been obtained using RAY, MVDOM and SOSVRT. This enabled us to produce benchmark results listed in the Appendix (see also more complete benchmark results (with $1^{\circ}$ viewing zenith angle (VZA) grid) at http://www.iup.uni-bremen. de/ alexk). MYSTIC gave equal results as discrete ordinate techniques within the inherent statistical noise of the Monte-Carlo method for molecular and aerosol scattering cases. The number of photons was $N=10^{8}$. However, the more accurate calculation of the fourth Stokes vector element for the aerosol scattering case requires more photons and, therefore, the results for the component $V$ derived with $N=10^{8}$ using MYSTIC differ from those obtained using SCIATRAN. This is not the case 
a
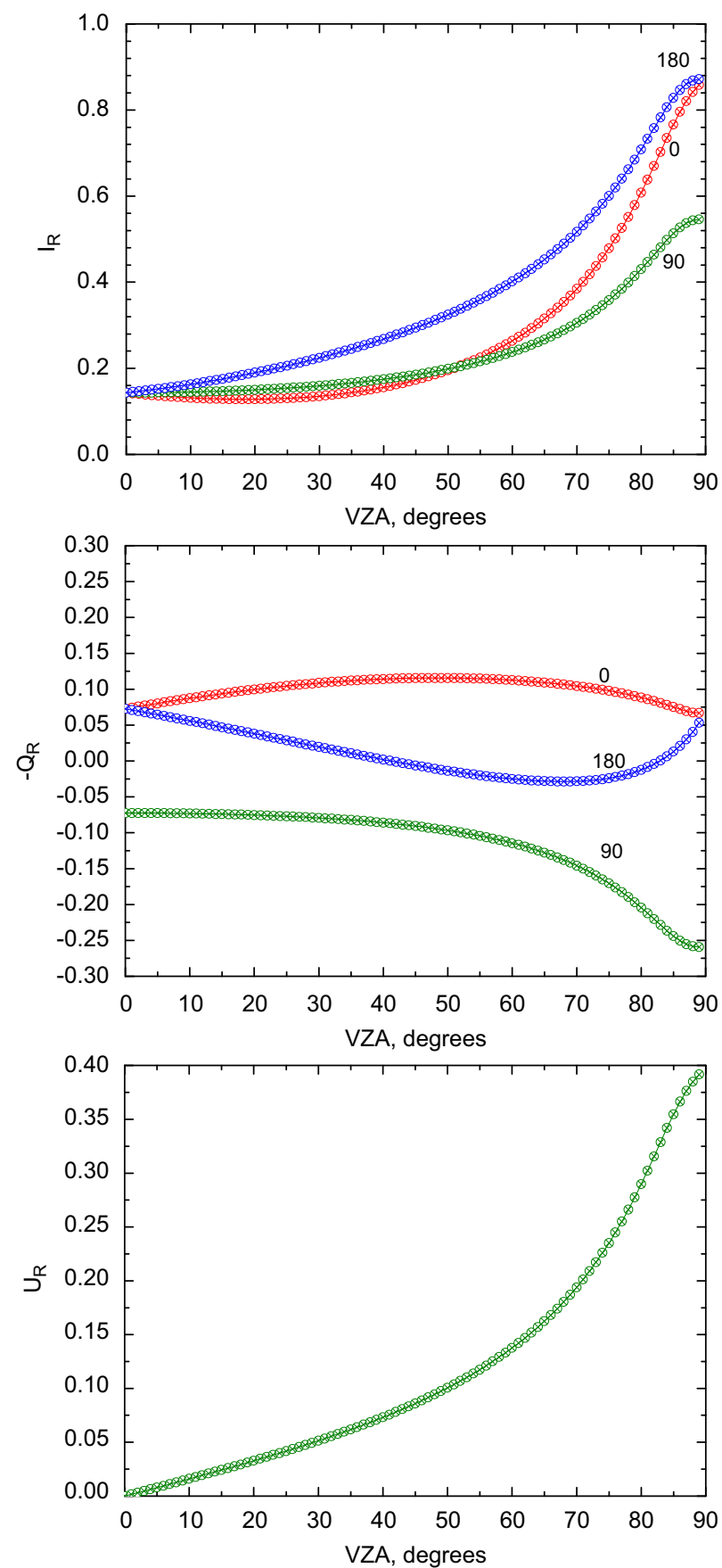

b
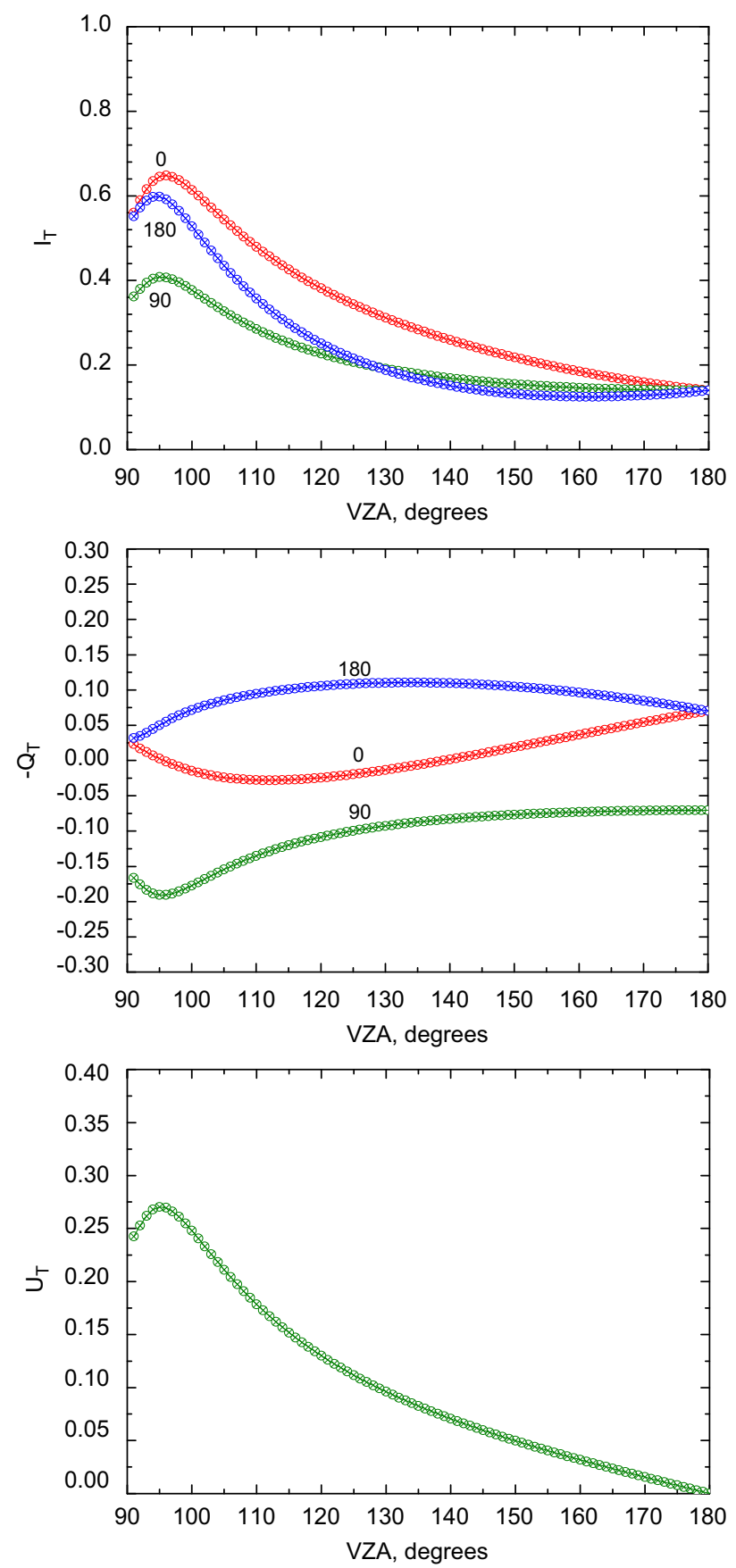

Fig. 2. (a) The normalized Stokes vector elements for the molecular scattering case in the reflected light (line-SCIATRAN, circles-Pstar, crosses-MYSTIC). The zenith incidence angle is $60^{\circ}$ and relative azimuths are $0^{\circ}, 90^{\circ}$, and $180^{\circ}$. Azimuths counter clock-wise. The third Stokes parameter vanishes at $\phi=0^{\circ}, 180^{\circ}$ and (b) The same as in (a) except for the transmitted light.

for the components $(I, Q U)$ usually used in remote sensing of terrestrial atmosphere. This note on the problem with the calculation of the fourth Stokes vector component (as $V \rightarrow 0$ ) is also relevant to 3DMCPOL. Otherwise, 3DMCPOL produces very accurate results for the molecular and aerosol scattering cases both in reflected and transmitted light.

The case of cloud scattering is calculated accurately for all Stokes parameters by SCIATRAN, SOSVRT, MVDOM and Pstar. MYSTIC produces accurate results for $I, Q$ and $U$ for 

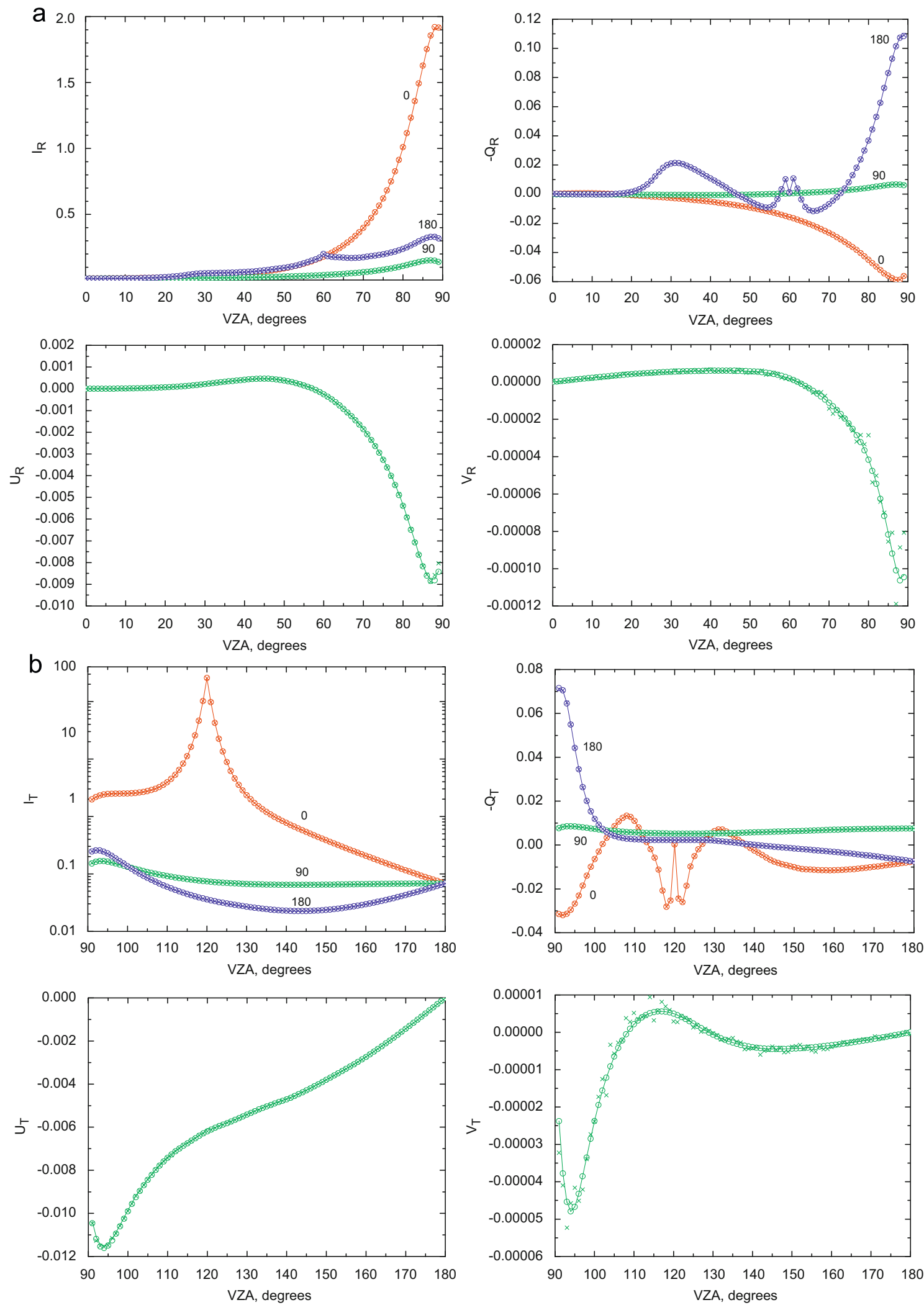

Fig. 3. (a) The same as in Fig. 2a except for the aerosol layer and (b) the same as in Fig. 2b except for the aerosol layer. 

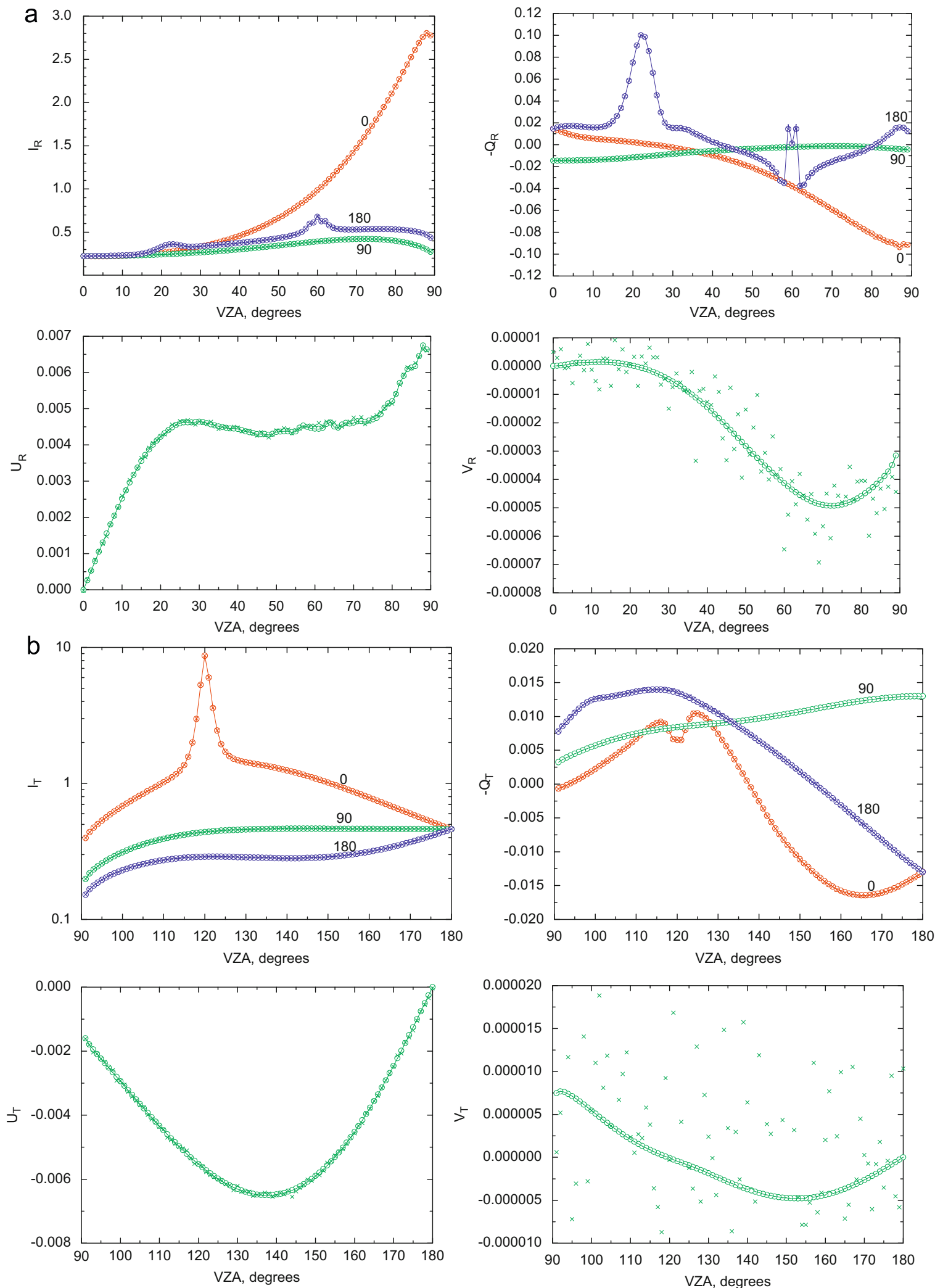

Fig. 4. (a) The same as in Fig. 2a except for the cloud layer and (b) the same as in Fig. 2b except for the cloud layer. 
the cloud phase function. The time of calculations for the discrete ordinate codes is almost not influenced by the optical thickness of the cloud. This is not the case for MYSTIC, here the time of calculations increases considerably with cloud optical thickness (especially for the case of nonabsorbing media considered in this paper). The component $V$ requires at least $10^{10}$ photons for the correct characterization and the results derived with $N=10^{8}$ are characterized by a considerable noise (see Fig. 4). $3 \mathrm{DMCPOL}$ is not optimized for the case of a cloudy atmosphere and, therefore, the corresponding results are not shown here. Although, as reported by corresponding teams, the results in reflected light for the components $(I, Q, U)$ derived using 3DMCPOL are similar to those shown in Fig. 4a. Some problems arise only for the component $V$ and also for transmitted light characteristics.

The $R A Y$ code gives less than $1 \%$ difference with SCIATRAN for the transmitted and reflected light intensities in the case of aerosol and molecular scattering cases. Errors grow, if clouds are under consideration. As it was mentioned above the used RAY version (Win32 application that runs on an ordinary PC) limits the order of Gauss quadratures to 81. And even in this case the maximal errors in calculation of the parameters $U$ and $Q$ (glory area) do not exceed 3\%.

The value of $V$ is equal to zero for Rayleigh scattering. $R A Y$ and SOSVRT can be used for the calculation of the fourth Stokes parameter and the difference to SCIATRAN is below $1 \%$ for the aerosol case then. However, the differences in the values of the fourth Stokes parameter (as compared to SCIATRAN) are larger than 5\% for RAY, SOSVRT, and Monte-Carlo codes in the case of cloud. The differences are smaller for Pstar $(0.2 \%$ and $0.5 \%$ for aerosol and cloud, respectively) and MVDOM (smaller than $1 \%$ for aerosol and $2 \%$ for cloud). Note that 100 streams was used for aerosol and 300 streams for clouds in the framework of MVDOM. This is smaller as compared to SCIATRAN (240 and 360 streams, respectively). MVDOM takes into account all expansion coefficients of the phase matrix.

The differences in the values of the fourth Stokes parameter for an aerosol layer in the case of Monte-Carlo

\section{Table 1}

The relative differences (in percent) of results for the first Stokes vector component (light intensity) derived from various codes as compared to SCIATRAN $\left(\vartheta_{0}=60^{\circ}, \vartheta=0-89^{\circ}, \phi=0^{\circ}, 90^{\circ}, 180^{\circ}\right)$ separately for Rayleigh scattering, aerosol, and cloud (both for reflected and transmitted light). The maximal relative differences over the studied viewing geometries are given.

\begin{tabular}{|c|c|c|c|c|}
\hline Case & Rayleigh & Aerosol & Cloud & Comments \\
\hline Pstar & 0.005 & 0.09 & 0.6 & $\begin{array}{l}\text { Cloud: for reflected light, and also for transmitted light and not very oblique } \\
\text { observation angles differences are below } 0.2 \%\end{array}$ \\
\hline MYSTIC & 0.05 & 0.6 & 0.4 & $\begin{array}{l}\text { Aerosol and cloud: for most of angles differences are below } 0.2 \% \\
\text { Aerosol: the differences go up to } 1.2 \% \text { at oblique observation angles }\end{array}$ \\
\hline 3DMCPOL & 0.25 & 3.0 & 5.0 & $\begin{array}{l}\text { Rayleigh: } 0.15 \% \text {, if } 89^{\circ} \text { angle is avoided; } \\
\text { Aerosol: } 1.5 \% \text {, if the angles above } 80^{\circ} \text { are avoided; } \\
\text { Cloud: the errors are larger in the vicinity of the forward peak; for most of cases } \\
\text { differences are below } 5 \%\end{array}$ \\
\hline$R A Y$ & 0.003 & 0.8 & 3.0 & $\begin{array}{l}\text { Aerosol: differences are below } 0.2 \% \text { if the rainbow region is excluded; } \\
\text { Cloud: differences can be further reduced taking more streams }\end{array}$ \\
\hline SOSVRT & 0.0005 & 0.5 & 0.2 & $\begin{array}{l}\text { The differences are below } 0.3 \% \text { for aerosol and } 0.03 \% \text { for clouds if the small } \\
\text { scattering region (transmitted light close to the direction of the direct beam) is } \\
\text { excluded }\end{array}$ \\
\hline MVDOM & 0.1 & 0.8 & 0.3 & $\begin{array}{l}\text { Rayleigh: differences increase up to } 0.2 \% \text { for the transmitted light and the oblique } \\
\text { observation (for } \vartheta \text { larger than } 80^{\circ} \text { ) }\end{array}$ \\
\hline
\end{tabular}

Table 2

The relative differences (in percent) of results for the second and third Stokes vector component derived from various codes as compared to SCIATRAN $\left(\vartheta_{0}=60^{\circ}, \vartheta=0-89^{\circ}, \phi=0,90,180^{\circ}\right)$ separately for Rayleigh scattering, aerosol, and cloud (both for reflected and transmitted light). The maximal relative differences (in percent) over the studied viewing geometries are given. The regions, where $U$ and $Q$ are close to zero are excluded because the relative difference is not defined then.

\begin{tabular}{|c|c|c|c|c|c|c|}
\hline Code & $\delta Q / Q$ (Rayleigh) & $\delta Q / Q($ aerosol) & $\delta Q / Q$ (cloud) & $\delta U / U$ (Rayleigh) & $\delta U / U$ (aerosol) & $\delta U / U$ (cloud) \\
\hline Pstar & 0.2 & 0.2 & 0.5 & 0.005 & 0.2 & 0.2 \\
\hline MYSTIC & 0.5 & 1.0 & 2.0 & 0.4 & 1.0 & 2.0 \\
\hline 3DMCPOL & 1.0 & 4.0 & $>5$ & 0.4 & 5.0 & $>5$ \\
\hline$R A Y$ & 0.06 & 1.0 & 1.0 & 0.004 & 1.0 & 1.0 \\
\hline SOSVRT & 0.2 & 0.8 & 0.5 & 0.005 & 0.5 & 0.2 \\
\hline MVDOM & 0.2 & 2.0 & 2.0 & 0.2 & 1.0 & 0.4 \\
\hline
\end{tabular}


codes studied in this work are larger than $5 \%$. This is related to the fact that the corresponding values are very small (below 0.00005 for aerosol and 0.000008 for cloud). The Monte-Carlo noise obtained with $10^{8}$ photons is larger than the value of $V$.

The summary of relative differences of the results for intensity obtained from different codes $(x)$ as compared to SCIATRAN $(s)(\{x / s-1\}$ in percent) is given in Table 1 for all discussed codes. It follows that Pstar produces the closest results as compared to SCIATRAN, which comes at no surprise because the general theoretical scheme (discrete ordinates) followed in both codes is the same. The next code of high performance is SOSVRT with the results similar to those derived from SCIATRAN and Pstar. The summary of the results for $Q$ und $U$ is given in Table 2 , where the results in the vicinity of points $Q=0$ and $U=0$ are avoided because then the relative difference tends to infinity (due to the definition of the relative difference). For MYSTIC the relative differences given in the tables are dominated by results obtained for observation angles with relatively small radiance values. The differences between MYSTIC and SCIATRAN are in all cases smaller than the Monte-Carlo noise.

In addition to inter-comparisons presented in this paper, authors of this work performed comparisons with many other similar studies and tables (see, e.g., [7-9,11]). In all cases the excellent agreement was found. The superiority of this inter-comparison study (see Figs. 2-4) is in fact due to high angular resolution of calculations $\left(1^{\circ}\right)$. So the errors of the codes in the vicinity of fine angular structures (e.g., glories, rainbows, etc.) can be studied in detail. All codes can be obtained from the authors using the links given in Appendix.

\section{Acknowledgements}

The work of Alexander Kokhanovsky was supported by the German Science Foundation (DFG) via the Project B-688-18-1 “Terra”. Claudia Emde's work was performed within the ESA project ESASLight, ESTEC contract AO/1$5433 / 07 / \mathrm{NL} / \mathrm{HE}$. The work of Yoshifumi Ota was performed as a part of Greenhouse gases observing satellite (GOSAT) project of National Institute for Environmental Studies (NIES), which collaborates with GOSAT research announcement program conducted by A. Kokhanovsky. Minzheng Duan's work was supported by the National Basic Research Program of China, No. 2006CB403702. We thank M. Mishchenko for providing the Mie code spher.f used in this study. The authors are grateful to three anonymous reviewers for their considerable help and important comments.

\section{Appendix. Benchmark results}

See Tables A1-A7.

Table A1

Radiative characteristics of a pure molecular atmosphere at $\vartheta_{0}=60^{\circ} \tau_{0}=0.3262, \omega_{0}=1$, and the values of the relative azimuth (from top to bottom in the cells of the table) $\phi=0^{\circ}, 90^{\circ}, 180^{\circ}$. The relative azimuth equal to $180^{\circ}$ corresponds to the backscattering geometry at $\vartheta=\vartheta_{0}$. The underlying surface albedo is equal to zero. 60 streams (in a hemisphere) have been used in calculations using SCIATRAN. If 100 streams are used than only the seventh significant figure is affected for some geometries; otherwise the result stays the same as given here. This is true for the third Stokes parameter as well. So the results shown in this table are correct at least for the first six digits. In this table and also in the tables below, the viewing zenith angle (VZA) is counted from the outer normal to the layer both for transmitted and reflected light characteristics. For the transmitted light, the VZA given in this table and also in other tables shown below differs from the angle in Figs. $2 \mathrm{~b}, 3 \mathrm{~b}, 4 \mathrm{~b}$ (e.g., the case $0^{\circ}$ in tables for the transmitted light corresponds to the angle $180^{\circ}$ shown in the figures and it follows: VZA $=180^{\circ}-\vartheta$, where $\vartheta$ is shown in Figs. $2 \mathrm{~b}, 3 \mathrm{~b}, 4 \mathrm{~b}$ ).

\begin{tabular}{|c|c|c|c|c|}
\hline VZA (deg.) & $I_{R}$ & $-Q_{R}$ & $I_{T}$ & $-Q_{T}$ \\
\hline 0 & $\begin{array}{l}0.1433981 E+00 \\
0.1433981 E+00 \\
0.1433981 E+00\end{array}$ & $\begin{array}{r}0.7253129 E-01 \\
-0.7253129 E-01 \\
0.7253129 E-01\end{array}$ & $\begin{array}{l}0.1396923 E+00 \\
0.1396923 E+00 \\
0.1396923 E+00\end{array}$ & $\begin{array}{r}0.7040628 \mathrm{E}-01 \\
-0.7040628 \mathrm{E}-01 \\
0.7040628 \mathrm{E}-01\end{array}$ \\
\hline 20 & $\begin{array}{l}0.1281929 E+00 \\
0.1501054 E+00 \\
0.1899151 E+00\end{array}$ & $\begin{array}{r}0.9953042 E-01 \\
-0.7537969 E-01 \\
0.3780822 E-01\end{array}$ & $\begin{array}{l}0.1846301 E+00 \\
0.1460187 E+00 \\
0.1247472 E+00\end{array}$ & $\begin{array}{r}0.3655640 \mathrm{E}-01 \\
-0.7306526 \mathrm{E}-01 \\
0.9643928 \mathrm{E}-01\end{array}$ \\
\hline 40 & $\begin{array}{l}0.1560517 E+00 \\
0.1746397 E+00 \\
0.2682317 E+00\end{array}$ & $\begin{array}{r}0.1140612 E+00 \\
-0.8605730 E-01 \\
0.1881170 E-02\end{array}$ & $\begin{array}{l}0.2590920 E+00 \\
0.1689676 E+00 \\
0.1509949 E+00\end{array}$ & $\begin{array}{r}0.1549839 \mathrm{E}-02 \\
-0.8295291 \mathrm{E}-01 \\
0.1096469 \mathrm{E}+00\end{array}$ \\
\hline 60 & $\begin{array}{l}0.2641460 \mathrm{E}+00 \\
0.2379419 \mathrm{E}+00 \\
0.4017650 \mathrm{E}+00\end{array}$ & $\begin{array}{r}0.1125426 E+00 \\
-0.1146228 E+00 \\
-0.2507640 E-01\end{array}$ & $\begin{array}{l}0.3811575 E+00 \\
0.2265788 E+00 \\
0.2511095 E+00\end{array}$ & $\begin{array}{r}-0.2444004 \mathrm{E}-01 \\
-0.1086739 \mathrm{E}+00 \\
0.1056079 \mathrm{E}+00\end{array}$ \\
\hline 80 & $\begin{array}{l}0.6078904 E+00 \\
0.4301002 E+00 \\
0.7084867 E+00\end{array}$ & $\begin{array}{r}0.8824163 E-01 \\
-0.2043280 E+00 \\
-0.1235467 E-01\end{array}$ & $\begin{array}{l}0.6138623 E+00 \\
0.3769405 E+00 \\
0.5277571 E+00\end{array}$ & $\begin{array}{r}-0.1452666 \mathrm{E}-01 \\
-0.1773986 \mathrm{E}+00 \\
0.7157849 \mathrm{E}-01\end{array}$ \\
\hline 89 & $\begin{array}{l}0.8581319 E+00 \\
0.5458706 E+00 \\
0.8718045 E+00\end{array}$ & $\begin{array}{r}0.6684238 E-01 \\
-0.2592859 E+00 \\
0.5316980 E-01\end{array}$ & $\begin{array}{l}0.5599639 E+00 \\
0.3617355 E+00 \\
0.5515021 E+00\end{array}$ & $\begin{array}{r}0.2324266 \mathrm{E}-01 \\
-0.1666421 \mathrm{E}+00 \\
0.3170448 \mathrm{E}-01\end{array}$ \\
\hline
\end{tabular}




\section{Table A2}

Radiative characteristics of an aerosol layer at $\vartheta_{0}=60^{\circ} \tau_{0}=0.3262, \omega_{0}=1$, and the values of the relative azimuth (from top to bottom in the cells of the table) $\phi=0^{\circ}, 90^{\circ}, 180^{\circ} .480$ streams (in a hemisphere) have been used in calculations using SCIATRAN. The underlying surface albedo is equal to zero. In most cases SCIATRAN and Pstar give at least the same two significant figures for the aerosol and cloud scattering.

\begin{tabular}{|c|c|c|c|c|}
\hline VZA (deg.) & $I_{R}$ & $-Q_{R}$ & $I_{T}$ & $-Q_{T}$ \\
\hline 0 & $\begin{array}{l}0.1436885 E-01 \\
0.1436885 E-01 \\
0.1436885 E-01\end{array}$ & $\begin{array}{r}0.1968219 E-03 \\
-0.1968219 E-03 \\
0.1968219 E-03\end{array}$ & $\begin{array}{l}0.6809063 E-01 \\
0.6809063 E-01 \\
0.6809063 E-01\end{array}$ & $\begin{array}{r}-0.7518252 \mathrm{E}-02 \\
0.7518252 \mathrm{E}-02 \\
-0.7518252 \mathrm{E}-02\end{array}$ \\
\hline 20 & $\begin{array}{l}0.2091866 \mathrm{E}-01 \\
0.1592941 \mathrm{E}-01 \\
0.2438049 \mathrm{E}-01\end{array}$ & $\begin{array}{r}-0.6995574 \mathrm{E}-03 \\
-0.3235966 \mathrm{E}-03 \\
0.1556273 \mathrm{E}-02\end{array}$ & $\begin{array}{l}0.2068393 E+00 \\
0.6628790 E-01 \\
0.2959081 E-01\end{array}$ & $\begin{array}{r}-0.1153345 E-01 \\
0.6934062 E-02 \\
-0.3156305 E-02\end{array}$ \\
\hline 40 & $\begin{array}{l}0.4936534 \mathrm{E}-01 \\
0.2230096 \mathrm{E}-01 \\
0.6320488 \mathrm{E}-01\end{array}$ & $\begin{array}{r}-0.5210978 \mathrm{E}-02 \\
0.5724128 \mathrm{E}-03 \\
0.1077220 \mathrm{E}-01\end{array}$ & $\begin{array}{l}0.7707225 E+00 \\
0.6451028 E-01 \\
0.2285936 E-01\end{array}$ & $\begin{array}{r}-0.2496497 \mathrm{E}-02 \\
0.5735927 \mathrm{E}-02 \\
0.9314070 \mathrm{E}-04\end{array}$ \\
\hline 60 & $\begin{array}{l}0.1801996 E+00 \\
0.4006522 E-01 \\
0.1995878 E+00\end{array}$ & $\begin{array}{r}-0.1575206 \mathrm{E}-01 \\
-0.4402361 \mathrm{E}-03 \\
0.1224099 \mathrm{E}-02\end{array}$ & $\begin{array}{l}0.2539210 E+03 \\
0.7393476 E-01 \\
0.3589802 E-01\end{array}$ & $\begin{array}{l}0.2572585 E-03 \\
0.5195650 E-02 \\
0.2253185 E-02\end{array}$ \\
\hline 80 & $\begin{array}{l}0.1009490 E+01 \\
0.1092879 E+00 \\
0.2390010 E+00\end{array}$ & $\begin{array}{r}-0.4498639 \mathrm{E}-01 \\
0.4325278 \mathrm{E}-02 \\
0.3671108 \mathrm{E}-01\end{array}$ & $\begin{array}{l}0.2509366 E+01 \\
0.1328353 E+00 \\
0.1357944 E+00\end{array}$ & $\begin{array}{r}-0.6418075 \mathrm{E}-02 \\
0.7285748 \mathrm{E}-02 \\
0.1182277 \mathrm{E}-01\end{array}$ \\
\hline 89 & $\begin{array}{l}0.1907928 E+01 \\
0.1390972 E+00 \\
0.3164781 E+00\end{array}$ & $\begin{array}{c}-0.5598186 \mathrm{E}-01 \\
0.6048002 \mathrm{E}-02 \\
0.1085128 \mathrm{E}+00\end{array}$ & $\begin{array}{l}0.1968607 E+01 \\
0.1514427 E+00 \\
0.2433105 E+00\end{array}$ & $\begin{array}{r}-0.3150199 \mathrm{E}-01 \\
0.7653060 \mathrm{E}-02 \\
0.7152042 \mathrm{E}-01\end{array}$ \\
\hline
\end{tabular}

\section{Table A3}

Radiative characteristics of a cloud layer at $\vartheta_{0}=60^{\circ} \tau_{0}=5.0, \omega_{0}=1$ and the values of the relative azimuth $\phi=0^{\circ}, 90^{\circ}, 180^{\circ}$ (from top to bottom in the cells of the table). 360 streams (in a hemisphere) have been used in calculations using SCIATRAN. The underlying surface albedo is equal to zero.

\begin{tabular}{|c|c|c|c|c|}
\hline VZA (deg.) & $I_{R}$ & $-Q_{R}$ & $I_{T}$ & $-Q_{T}$ \\
\hline 0 & $\begin{array}{l}0.2230386 E+00 \\
0.2230386 E+00 \\
0.2230386 E+00\end{array}$ & $\begin{array}{r}0.1458306 \mathrm{E}-01 \\
-0.1458306 \mathrm{E}-01 \\
0.1458306 \mathrm{E}-01\end{array}$ & $\begin{array}{l}0.4626634 \mathrm{E}+00 \\
0.4626634 \mathrm{E}+00 \\
0.4626634 \mathrm{E}+00\end{array}$ & $\begin{array}{r}-0.1300066 \mathrm{E}-01 \\
0.1300066 \mathrm{E}-01 \\
-0.1300066 \mathrm{E}-01\end{array}$ \\
\hline 20 & $\begin{array}{l}0.2619955 E+00 \\
0.2416682 E+00 \\
0.3301769 E+00\end{array}$ & $\begin{array}{r}0.2192489 E-02 \\
-0.1164763 E-01 \\
0.7511787 E-01\end{array}$ & $\begin{array}{l}0.7871585 E+00 \\
0.4634229 E+00 \\
0.3147638 E+00\end{array}$ & $\begin{array}{r}-0.1575456 \mathrm{E}-01 \\
0.1180246 \mathrm{E}-01 \\
-0.3126918 \mathrm{E}-02\end{array}$ \\
\hline 40 & $\begin{array}{l}0.4587779 E+00 \\
0.3007135 E+00 \\
0.3761572 E+00\end{array}$ & $\begin{array}{r}-0.9805261 E-02 \\
-0.5759207 E-02 \\
0.2496259 E-02\end{array}$ & $\begin{array}{l}0.1248475 E+01 \\
0.4646386 E+00 \\
0.2823214 E+00\end{array}$ & $\begin{array}{r}-0.2553568 \mathrm{E}-02 \\
0.9685827 \mathrm{E}-02 \\
0.6388771 \mathrm{E}-02\end{array}$ \\
\hline 60 & $\begin{array}{l}0.9851353 E+00 \\
0.3921383 E+00 \\
0.6797278 E+00\end{array}$ & $\begin{array}{r}-0.3790092 E-01 \\
-0.2105265 E-02 \\
0.9801442 E-03\end{array}$ & $\begin{array}{l}0.8658228 E+01 \\
0.4388206 E+00 \\
0.2889342 E+00\end{array}$ & $\begin{array}{l}0.6463815 \mathrm{E}-02 \\
0.8307814 \mathrm{E}-02 \\
0.1341101 \mathrm{E}-01\end{array}$ \\
\hline 80 & $\begin{array}{l}0.2186265 E+01 \\
0.4003249 E+00 \\
0.5330462 E+00\end{array}$ & $\begin{array}{l}-0.8132911 \mathrm{E}-01 \\
-0.2089776 \mathrm{E}-02 \\
-0.2216694 \mathrm{E}-02\end{array}$ & $\begin{array}{l}0.6816960 E+00 \\
0.3124113 E+00 \\
0.2304723 E+00\end{array}$ & $\begin{array}{l}0.2234633 E-02 \\
0.5702380 E-02 \\
0.1257132 E-01\end{array}$ \\
\hline 89 & $\begin{array}{l}0.2776746 E+01 \\
0.2728933 E+00 \\
0.4380821 E+00\end{array}$ & $\begin{array}{r}-0.9160169 E-01 \\
-0.4403001 E-02 \\
0.1232541 E-01\end{array}$ & $\begin{array}{l}0.3971848 E+00 \\
0.1986522 E+00 \\
0.1522583 E+00\end{array}$ & $\begin{array}{r}-0.6617358 \mathrm{E}-03 \\
0.3243696 \mathrm{E}-02 \\
0.7785649 \mathrm{E}-02\end{array}$ \\
\hline
\end{tabular}

Table A4

The third normalized Stokes parameter for the reflected light at $\vartheta_{0}=60^{\circ}, \phi=90^{\circ}$. Other input parameters are the same as in the tables above.

\begin{tabular}{lll}
\hline VZA (deg.) & $U_{R}$ (Rayleigh) & $U_{R}$ (aerosol) \\
\hline 0 & 0.0 & 0.0 \\
20 & $0.3284170 \mathrm{E}-01$ & $0.6485331 \mathrm{E}-04$ \\
40 & $0.7322031 \mathrm{E}-01$ & $0.4139335 \mathrm{E}-03$ \\
60 & $0.1376191 \mathrm{E}+00$ & $0.4223818 \mathrm{E}-02$ \\
80 & $0.2896555 \mathrm{E}+00$ & $0.4437616 \mathrm{E}-02$ \\
89 & $0.3917105 \mathrm{E}+00$ & $-0.538693 \mathrm{E}-03$ \\
\hline
\end{tabular}


Table A5

The third normalized Stokes parameter for the transmitted light at $\vartheta_{0}=60^{\circ}, \phi=90^{\circ}$. Other parameters are as in the tables above.

\begin{tabular}{llcr}
\hline VZA (deg.) & $U_{T}$ (Rayleigh) & $U_{T}$ (aerosol) & $U_{T}$ (cloud) \\
\hline 0 & 0.0 & 0.0 & 0.0 \\
20 & $0.3186302 \mathrm{E}-01$ & $-0.2740344 \mathrm{E}-02$ & $-0.4523557 \mathrm{E}-02$ \\
40 & $0.7055535 \mathrm{E}-01$ & $-0.4707951 \mathrm{E}-02$ & $-0.6480793 \mathrm{E}-02$ \\
60 & $0.1300480 \mathrm{E}+00$ & $-0.6199702 \mathrm{E}-02$ & $-0.5535509 \mathrm{E}-02$ \\
80 & $0.2479299 \mathrm{E}+00$ & $-0.9904365 \mathrm{E}-02$ & $-0.2947084 \mathrm{E}-02$ \\
89 & $0.2424255 \mathrm{E}+00$ & $-0.1046631 \mathrm{E}-01$ & $-0.1604392 \mathrm{E}-02$ \\
\hline
\end{tabular}

\section{Table A6}

The fourth normalized Stokes parameter at $\vartheta_{0}=60^{\circ}, \phi=90^{\circ}, \tau_{0}=0.3262$ for aerosol and $\tau_{0}=5$ for a cloud both for reflected and transmitted light. Other parameters are as in the tables above.

\begin{tabular}{cccrr}
\hline VZA (deg.) & $V_{R}$ (aerosol) & \multicolumn{1}{l}{$V_{R}$ (cloud) } & \multicolumn{1}{c}{$V_{T}$ (aerosol) } & \multicolumn{1}{c}{$V_{T}$ (cloud) } \\
\hline 0 & 0 & 0 & 0 & 0 \\
20 & $0.4181747 \mathrm{E}-05$ & $0.3947605 \mathrm{E}-06$ & $-0.3480572 \mathrm{E}-05$ & $0.4382299 \mathrm{E}-05$ \\
40 & $0.6002111 \mathrm{E}-05$ & $-0.1453557 \mathrm{E}-04$ & $-0.3988220 \mathrm{E}-05$ & $0.3722931 \mathrm{E}-05$ \\
60 & $0.1781059 \mathrm{E}-05$ & $-0.4132179 \mathrm{E}-04$ & $0.4991464 \mathrm{E}-05$ & $0.5919457 \mathrm{E}-08$ \\
80 & $-0.4169772 \mathrm{E}-04$ & $-0.4582790 \mathrm{E}-04$ & $-0.2378265 \mathrm{E}-04$ & $-0.5441439 \mathrm{E}-05$ \\
89 & $-0.1045741 \mathrm{E}-03$ & $-0.3166770 \mathrm{E}-04$ & $-0.2380531 \mathrm{E}-04$ & $-0.7481497 \mathrm{E}-05$
\end{tabular}

Table A7

The list of used codes and corresponding links/e-mails.

\begin{tabular}{lll}
\hline No. & Code & Link/e-mail \\
\hline 1 & SCIATRAN & www.iup.physik.uni-bremen.de/sciatran/ \\
2 & Pstar & www.ccsr.u-tokyo.ac.jp/ clastr/ \\
3 & MVDOM & www.svet-mpei.org \\
4 & RAY & eleonor@light.basnet.by \\
5 & SOSVRT & dmz@mail.iap.ac.cn \\
6 & 3DMCPOL & cornet@loa.univ-lille1.fr \\
7 & MYSTIC & claudia.emde@lmu.de \\
\hline
\end{tabular}

\section{References}

[1] Fresnel A. Oeuvres completes. Paris, 1868.

[2] Hecht E. Optics. Amsterdam: Addison-Wesley, Longman; 2002.

[3] Chandrasekhar S. Radiative transfer. New York: Dover; 1960.

[4] Liou KN. An introduction to atmospheric radiation. New York: Academic Press; 2002.

[5] Mishchenko MI, Travis L. Electromagnetic scattering by nonspherical particles. In: Guzzi R, editor. Exploring the atmosphere by remote sensing techniques. Berlin: Springer; 2003. p. 7-127.

[6] Rozanov AA, Rozanov VV, Buchwitz M, Kokhanovsky AA, Burrows JP. SCIATRAN 2.0-a new radiative transfer model for geophysical applications in the $175-2400 \mathrm{~nm}$ spectral range. Adv Space Res 20051015-9.

[7] Garcia RDM, Siewert CE. A generalized spherical harmonics solution for radiative transfer models that include polarization effects. J Quant Spectrosc Radiat Transfer 1986;36:401-23.

[8] Garcia RDM, Siewert CE. The $F_{N}$ method for radiative transfer models that include polarization effects. J Quant Spectrosc Radiat Transfer 1989;41:117-45.

[9] Mishchenko MI. Reflection of polarized light by plane-parallel slabs containing randomly-oriented nonspherical particles. J Quant Spectrosc Radiat Transfer 1991;46:171-81.

[10] Wauben WMF, Hovenier JW. Polarized radiation of an atmosphere containing randomly-oriented spheroids. J Quant Spectrosc Radiat Transfer 1992;47:491-504.
[11] Natraj V, Li K-F, Yung YL. Rayleigh scattering in planetary atmospheres: corrected tables through accurate computation of $X$ and $Y$ functions. Astrophys J 2009;691:1909-20.

[12] Rozanov VV, Kokhanovsky AA. The solution of the vector radiative transfer equation using the discrete ordinates technique: selected applications. Atmos Res 2006;79:241-65.

[13] Siewert CE. A discrete-ordinates solution for radiative-transfer models that include polarization effects. J Quant Spectrosc Radiat Transfer 2000;64:227-54.

[14] Ota Y, Higurashi A, Nakajima T, Yokota T. Matrix formulations of radiative transfer including the polarization effect in a coupled atmosphere-ocean system. J Quant Spectrosc Radiat Transfer 2010;111:878-94.

[15] Nakajima T, Tanaka M. Matrix formulations for the transfer of solar radiation in a plane-parallel scattering atmosphere. J Quant Spectrosc Radiat Transfer 1986;35:13-21.

[16] Ruggaber A, Dlugi R, Nakajima T. Modelling radiation quantities and photolysis frequencies in the troposphere. J Atmos Chem 1994:18:171-210.

[17] Nakajima T, Tanaka M. Algorithms for radiative intensity calculations in moderately thick atmospheres using a truncation approximation. J Quant Spectrosc Radiat Transfer 1988;40:51-69.

[18] Zege EP, Chaikovskaya LI. New approach to the polarized radiative transfer problem. J Quant Spectrosc Radiat Transfer 1996;55:19-31.

[19] Zege EP, Katsev IL, Polonsky IN. Multicomponent approach to light propagation in clouds and mists. Appl Opt 1993;32:2803-12.

[20] Zege EP, Ivanov AP, Katsev IL. Image transfer through a scattering medium. Heidelberg: Springer-Verlag; 1991.

[21] Lenoble J. Radiative transfer in scattering and absorbing atmospheres: standard computational procedures. Hampton: A. Deepak Publishing; 1985.

[22] Coulson KL, Dave J V, Sekera Z. Tables related to radiation emerging from a planetary atmosphere with Rayleigh scattering. Berkeley: University of California Press; 1960.

[23] Katsev IL, Prikhach AS, Zege EP, Ivanov AP, Kokhanovsky AA. Iterative procedure for retrieval of spectral aerosol optical thickness and surface reflectance from satellite data using fast radiative transfer code and its application to MERIS measurements. In: Kokhanovsky AA, de Leeuw G, editors. Satellite aerosol remote sensing over land. Berlin: Springer-Praxis; 2009. p. 101-34.

[24] Tynes H, Kattawar GW, Zege EP, Katsev IL, Prikhach AS, Chaikovskaya LI. Monte Carlo and multicomponent approximation methods for vector radiative transfer by use of effective Mueller matrix calculations. Appl Opt 2001;40:400-12.

[25] Mayer B. Radiative transfer in the cloudy atmosphere. Eur Phys J Conf 2009;1:75-99. 
[26] Emde C, Mayer B. Simulation of solar radiation during a total solar eclipse: a challenge for radiative transfer. Atmos Chem Phys 2007;7:2259-70.

[27] Emde C, Buras R, Mayer B, Blumthaler M. The impact of aerosols on polarized sky radiance: model development, validation, and applications. Atmos Chem Phys 2010;10:383-96.

[28] Mayer B, Kylling A. Technical note: the libRadtran software package for radiative transfer calculations-description and examples of use. Atmos Chem Phys 2005;5:1855-77.

[29] Marchuk GI, Mikhailov GA, Nazaraliev MA. The Monte Carlo methods in atmospheric optics. Berlin: Springer; 1980.

[30] Evans KF, Marshak A. Numerical methods. In: Marshak A, Davis AB editors. 3D radiative transfer in cloud atmospheres. Berlin: Springer-Verlag; 2005. p. 243-81.

[31] Collins DG, Blättner WG, Wells MB, Horak HG. Backward Monte Carlo calculations of the polarization characteristics of the radiation emerging from spherical-shell atmospheres. Appl Opt 1972;11: 2684-96.

[32] Buras R. A rapid unbiased Monte Carlo method for lidar simulations. In: MOCA 2009, Montreal, oral presentation, 22 July 2009.

[33] Blumthaler M, Schallhart B, Schwarzmann M, McHenzie R Johnston P, Kotkamp M, et al. Spectral UV measurements of global irradiance, solar radiance, and actinic flux in New Zealand: intercomparison between instruments and model calculations. J Atmos Ocean Technol 2008;25:945-58.

[34] Cornet C, C-Labonnote L, Szczap F. Three-dimensional polarized Monte Carlo atmospheric radiative transfer model (3DMCPOL): 3D effects on polarized visible reflectances of a cirrus cloud. J Quant Spectrosc Radiat Transfer 2010;111:174-86.

[35] Cornet C, C-Labonnote L, Szczap F. 3DMCPOL: using a 3D radiative transfer Monte-Carlo model to assess radiative effects on polarized reflectances above cloud scenes. In: Kokhanovsky A, editor. Light scattering reviews, vol. 5, 2010, p. 81-104.
[36] Min Q, Duan M. A successive order of scattering model for solving vector radiative transfer in the atmosphere. J Quant Spectrosc Radiat Transfer 2004;87:243-59.

[37] Duan M, Min Q, Lu D. A polarized radiative transfer model based on successive order of scattering. Adv Atmos Sci 2010 doi:10.1007| s00376-009-9049-8.

[38] Siewert CE. On the phase matrix basic to the scattering of polarized light. Astron Astrophys 1982;109:195-200.

[39] Hovenier J. Symmetry relations for scattering of polarized light in a slab of randomly oriented particles. J Atmos Sci 1969;26: 488-99.

[40] Budak VP, Korkin SV. On the solution of a vectorial radiative transfer equation in an arbitrary three-dimensional turbid medium with anisotropic scattering. J Quant Spectrosc Radiat Transfer 2008;109:220-34.

[41] Budak VP, Sarmin SE. Solution of the radiation transfer equation by the method of spherical harmonics in the small-angle modification. Atmos Opt 1990;3:898-904.

[42] Astakhov IE, Budak VP, Lisitsin DV, Selivanov VA. Solution of the vector radiative transfer equation in the small angle approximation of the spherical harmonics method. Atmos Oceanic Opt 1994;7: 398-403.

[43] Plass GN, Kattawar GW, Catchings FE. Matrix operator theory of radiative transfer. 1: Rayleigh scattering. Appl Opt 1973;12:314-29.

[44] Sykes JB. Approximate integration of the equation of transfer. Month Not R Astron Soc 1951;111:378-86.

[45] Karp AH, Greenstadt J, Fillmore JA. Radiative transfer through an arbitrary thick scattering atmosphere. J Quant Spectrosc Radiat Transfer 1980;24:391-406.

[46] Mark C. The neutron density near a plane surface. Phys Rev $1947 ; 72: 558-64$

[47] Gelfand IM, Minlos RA, Shapiro ZYa. Representations of the rotation and Lorentz groups and their applications. Oxford: Pergamon Press; 1963. 